\title{
Effects of self-ligating brackets and other factors influencing orthodontic treatment outcomes: A prospective cohort study
}

Min-Ho Jung ${ }^{\mathrm{a}, \mathrm{b}}$

${ }^{a}$ Department of Orthodontics, Dental Research Institute and School of Dentistry, Seoul National University, Seoul, Korea

${ }^{\mathrm{b}}$ Private Practice, Seoul, Korea

\begin{abstract}
Objective: The purpose of this study was to evaluate the effects of self-ligating brackets (SBs) and other factors that influence orthodontic treatment outcomes. Methods: This two-armed cohort study included consecutively treated patients in a private practice. The patients were asked to choose between SBs and conventional brackets (CBs); if any patient did not have a preference, he or she was randomly allocated to the CB or SB group. All patients were treated using an identical archwire sequence. Evaluated parameters were as follows: treatment duration, number of bracket failures, poor oral hygiene, poor elastic wear, extraction, use of orthodontic mini-implants (OMI), OMl failure, American Board of Orthodontics (ABO) Discrepancy Index (DI), arch length discrepancy, and ABO Cast-Radiograph Evaluation (CRE) score. Stepwise regression analysis was performed to generate the equation for prediction of the CRE. Results: The final sample comprised 134 patients with an average age of 22.73 years. The average Dl, CRE, and treatment duration were 21.81, 14.25, and 28.63 months, respectively. Analysis of covariance showed a significant difference in CRE between the CB and SB groups after adjusting for the effects of confounding variables. Stepwise regression analysis using four variables, namely extraction, SB use, poor elastic wear, and additional appliance use, could explain only 25.2\% of the variance in the CRE. Conclusions: Although the CRE was significantly better for CBs than for SBs, the clinical significance of this result seems to be limited. Extraction, SB use, poor elastic wear, and additional appliance use may have significant effects on treatment outcomes.
\end{abstract}

[Korean J Orthod 2021;51(6):397-406]

Key words: Bracket, Cast-Radiograph Evaluation, Orthodontic Index, Compliance

Received February 2, 2021; Revised June 22, 2021; Accepted July 2, 2021.

Corresponding author: Min-Ho Jung.

Private Practice, 3rd Fl., Taenam bldg, 40, Jamwon-ro 3-gil, Seocho-gu, Seoul 06510, Korea.

Tel +82-2-599-4001 e-mail fortit@chol.com

How to cite this article: Jung MH. Effects of self-ligating brackets and other factors influencing orthodontic treatment outcomes: A prospective cohort study. Korean J Orthod 2021;51:397-406.

(C) 2021 The Korean Association of Orthodontists.

This is an Open Access article distributed under the terms of the Creative Commons Attribution Non-Commercial License (http://creativecommons.org/licenses/by-nc/4.0) which permits unrestricted non-commercial use, distribution, and reproduction in any medium, provided the original work is properly cited. 


\section{INTRODUCTION}

The desire to improve treatment outcomes is common among orthodontists. Assessment of the quality of orthodontic treatment generally includes evaluation of posttreatment records such as study casts and panoramic radiographs. In 1995, the American Board of Orthodontics (ABO) developed the Cast-Radiograph Evaluation (CRE) system for scoring dental casts and panoramic radiographs for the precise clinical evaluation of orthodontic treatment outcomes; since then, this evaluation tool has been improved and modified through field tests.'

The ABO CRE comprises eight criteria: alignment, marginal ridges, buccolingual inclination, occlusal relationships, occlusal contacts, overjet, interproximal contacts, and root angulation. Other indices such as the Index of Orthodontic Treatment Need; Index of Complexity, Outcome, and Need; and Peer Assessment Rating Index are also used for evaluating treatment outcomes, but CRE is the most accurate and stringent method for evaluating treatment outcomes. ${ }^{2}$

Fixed appliances are a basic tool in comprehensive orthodontic treatment and allow three-dimensional control of tooth movement. Various efforts have been made to create more effective and efficient brackets to improve the quality of treatment over several years.

Self-ligating brackets (SBs) were introduced with the expectation that elimination of ligature ties would produce a friction-free environment and permit better sliding mechanics. However, systematic reviews showed that the treatment efficiency or effectiveness of SBs was not superior to that of conventional brackets (CBs)., ${ }^{3,4}$

If an orthodontist spends more time and effort to achieve better occlusion, better treatment outcomes may be obtained. Given that SBs can reduce the chair time, ${ }^{4}$ their use may lead to better treatment results because the clinician would have more time to evaluate and adjust the occlusion. On the other hand, some researchers argue that SBs have a complex structure, which results in a larger bracket volume and a longer distance between the tooth surface and archwire; therefore, accurate control of tooth movement is more difficult with SBs than with CBs (Figure 1). ${ }^{5}$ However, no studies have made such comparisons between CBs and SBs.

Extractions may affect the treatment quality. A recent systematic review showed that a 10\% increase in the extraction rate may decrease the CRE score by 0.7 points, which indicates a better occlusal outcome. ${ }^{6}$ However, most studies included in that review were retrospective, and additional studies are needed to validate the findings.

Although many studies have shown that patient cooperation can affect the treatment duration, ${ }^{7,8}$ few studies have investigated the association between patient cooperation and treatment outcomes.

Orthodontic mini-implants (OMls) are often used in cases involving difficult tooth movement, such as intrusion or asymmetric movement; therefore, cases where OMls are used may show worse treatment outcomes. However, prediction of the effects of OMls is difficult because they can provide the required anchorage without requiring patient cooperation. Considering that OMls have recently gained popularity, no study has evaluated the effects of OMl-related factors on treatment outcomes.

The influence of case complexity on treatment outcomes remains controversial. While some studies have shown no association, ${ }^{9,10}$ others have reported a significant correlation. ${ }^{11-13}$ In some clinical settings, simple cases are mostly treated by general practitioners, and orthodontists primarily treat difficult cases. Under these conditions, the average case complexity is relatively severe, so the extraction rate and frequency of OMl use
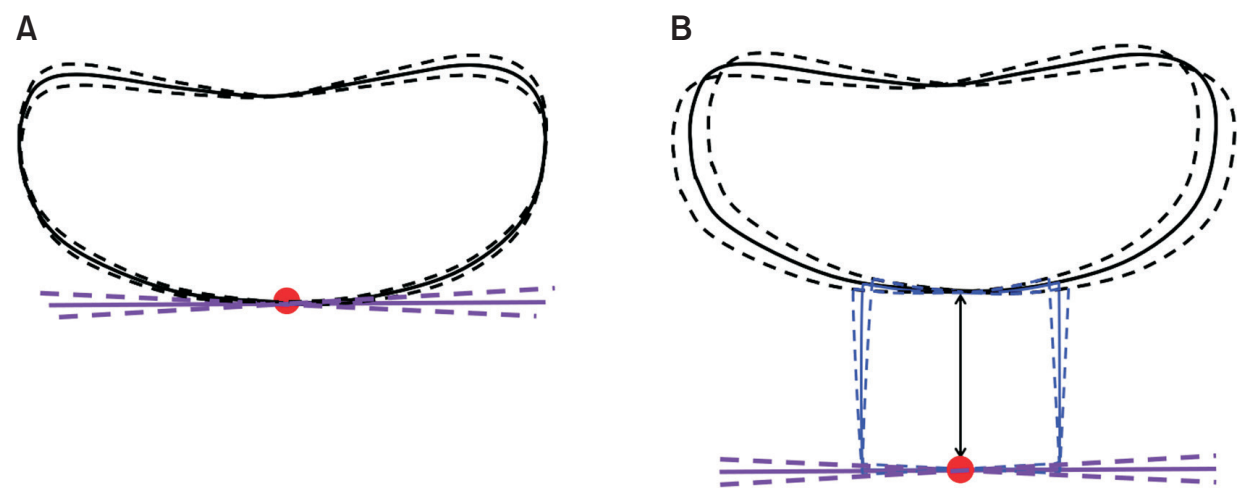

Figure 1. The size of a bracket and tooth control. A, Because of the "play," the tooth cannot be perfectly controlled even if the point of force application is located on the tooth surface. B, If the size of the bracket is large and the tooth surface is further away from the slot base, the inaccuracy of tooth control increases.

Red circle, point of force application; Purple line, archwire; Light blue rectangle, bracket. 
will be high.

Considering all the abovementioned factors, the goals of this study were to compare treatment outcomes between SBs and CBs and identify factors that affect treatment outcomes in clinical settings with a relatively severe average case complexity.

\section{MATERIALS AND METHODS}

The subjects were recruited from a sample of consecutive patients who began receiving orthodontic treatment in a single private practice between March 2008 and August 2009. More than $60 \%$ of patients visiting this private clinic select ceramic brackets; therefore, only patients treated with ceramic brackets were included in this study. The inclusion criteria were as follows: (1) permanent dentition, (2) no previous orthodontic treatment, and (3) need for comprehensive treatment. The exclusion criteria were as follows: (1) presence of tooth impaction (except the third molars), (2) requirement of jaw surgery, (3) presence of craniofacial anomalies, and (4) a complex medical or dental history. All the participants were allocated to a CB or an SB group. In the SB group, 0.022" Clippy-C (MBT prescription, Tomy, Tokyo, Japan) brackets were bonded. In the CB group, 0.022" Clarity (MBT prescription, 3M Unitek, Monrovia, CA, USA) brackets were bonded. The entire treatment procedure, measurements, and analyses were performed by a single investigator.

This study was approved by the Institutional Review Board of Seoul National University Dental Hospital (CR109008). All patients and parents received written and verbal information regarding the study, and informed consent was obtained in accordance with the Declaration of Helsinki.

Originally, this study was designed to investigate factors influencing the treatment duration and treatment outcomes. The sample size was calculated on the basis of the CRE score for fixed appliance treatment from a previous study ${ }^{13}$ and the treatment duration data from another ongoing study. ${ }^{14}$ Fortunately, both calculation methods yielded the same sample size ( 53 patients per group) to show a $20 \%$ difference with a $90 \%$ power at $\alpha$
$=0.05$. Assuming a 10\% drop-out rate, 59 patients were needed in each group. Considering the 20\% betweengroup difference that could occur during the allocation process (bracket selection by patients), it was decided that $\geq 133$ patients were required for this study.

Typodonts (Figure 2) with two types of brackets were presented to the patients. After differences in the wireholding mechanism and bracket shape were explained to them, the patients were asked to choose one of the two brackets. If a patient did not have a preference, the type of bracket was randomly selected through a coin toss.

The brackets were bonded by using conventional adhesive (Transbond XT; 3M Unitek). Leveling progressed with a predetermined archwire sequence: 014" nickeltitanium (NiTi), 016" NiTi, $018^{\prime \prime} \mathrm{NiTi}, 016 \times 022^{\prime \prime} \mathrm{NiTi}$, $019 \times 025^{\prime \prime} \mathrm{NiTi}$, and $019 \times 025^{\prime \prime}$ stainless steel. Each subject was reviewed at approximately 4-week intervals. Where required, OMls (Mplant U2; Biomaterials Korea Inc., Seoul, Korea) were placed during the leveling stage.

The measured variables were as follows.

- ABO Discrepancy Index (DI) and CRE score

- Irregularity index and arch length discrepancy (ALD)

- Extractions (or previously extracted tooth space)

- Bracket failure, bracket fracture, poor elastic wear, missed appointments, and poor oral hygiene

- OMI use, OMl failure, and additional appliance use

To examine the effects of bond failure and OMl failure on treatment outcomes, all cases of bond failure and $\mathrm{OMl}$ failure, including those with rebonded brackets and repositioned OMls, were recorded. Wing fracture is a concern with ceramic bracket use..$^{15}$ If the bracket wing was fractured and rotational control became difficult, the event was recorded and a new bracket was bonded. Intermaxillary elastics were prescribed at the finishing stage for all patients, and oral hygiene was evaluated using the Modified Plaque Index at each visit. The original Plaque Index evaluates the gingival area on four surfaces (buccal, lingual, mesial, and distal) of six teeth (\#12, 16, 24, 32, 36, and 44; 1S0), with the assigned score ranging from 0 to $3 .^{16}$ The Modified Plaque Index used in this study evaluated the gingival area on the labial surface of 12 anterior teeth, assigning a score from 0 to 3. If four or more teeth scored more than
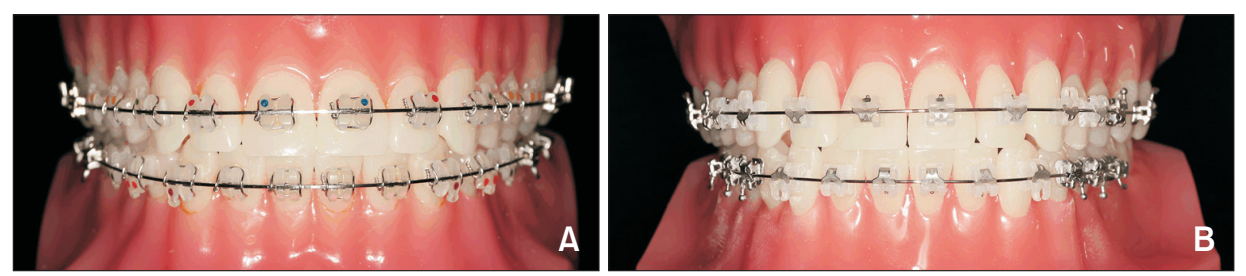

Figure 2. Typodonts with two types of brackets. A, Conventional bracket (Clarity ${ }^{\mathrm{TM}}$; MBT prescription, $3 \mathrm{M}$ Unitek, Monrovia, CA, USA). B, Self-ligating bracket (Clippy- $\mathrm{C}^{\mathrm{TM}}$; MBT prescription, Tomy, Tokyo, Japan). 
two points (plaque is visible with or without air drying), oral hygiene was judged to be poor. The use of appliances other than brackets (e.g., headgear, rapid palatal expander, Forsus appliance) was recorded and used as a confounding variable.

In this study, the investigator could not be blinded to the bracket type during treatment. After debonding, all identifiable information of the patient was removed from the casts and $\mathrm{X}$-rays, and a random identification number was assigned at the administrative office to minimize bias during analysis. All lateral cephalograms were traced and digitized by the same investigator (MHJ).

\section{Statistical analysis}

SPSS software (version 17.0; SPSS Inc., Chicago, IL, USA) was used for all statistical analyses. For evaluation of the baseline characteristics of the sample and confounding variables, descriptive analyses were performed. After the square root of the CRE score and logarithm of the treatment duration were obtained, normality was confirmed by the Shapiro-Wilk test.
The effects of nominal variables on CRE were analyzed using an independent $t$-test, while the effects of continuous variables were evaluated by correlation analysis. Using significant variables in the $t$-test and correlation analysis, analysis of covariance (ANCOVA) was performed to compare the CRE score between the two groups. Stepwise regression analysis was used for prediction of the CRE score. The Mann-Whitney $U$ test was also used to determine differences in each CRE criterion between the two bracket systems.

For the assessment of intraexaminer reliability, ABO DI, ALD, and CRE were measured again after a 4-week interval for 28 randomly selected cases, and the intraclass correlation coefficient (ICC) based on a two-way mixed effect model was calculated. Because all measurements were performed by a single investigator, interexaminer reliability was not evaluated.

\section{RESULTS}

During the study period, 321 patients started orth-

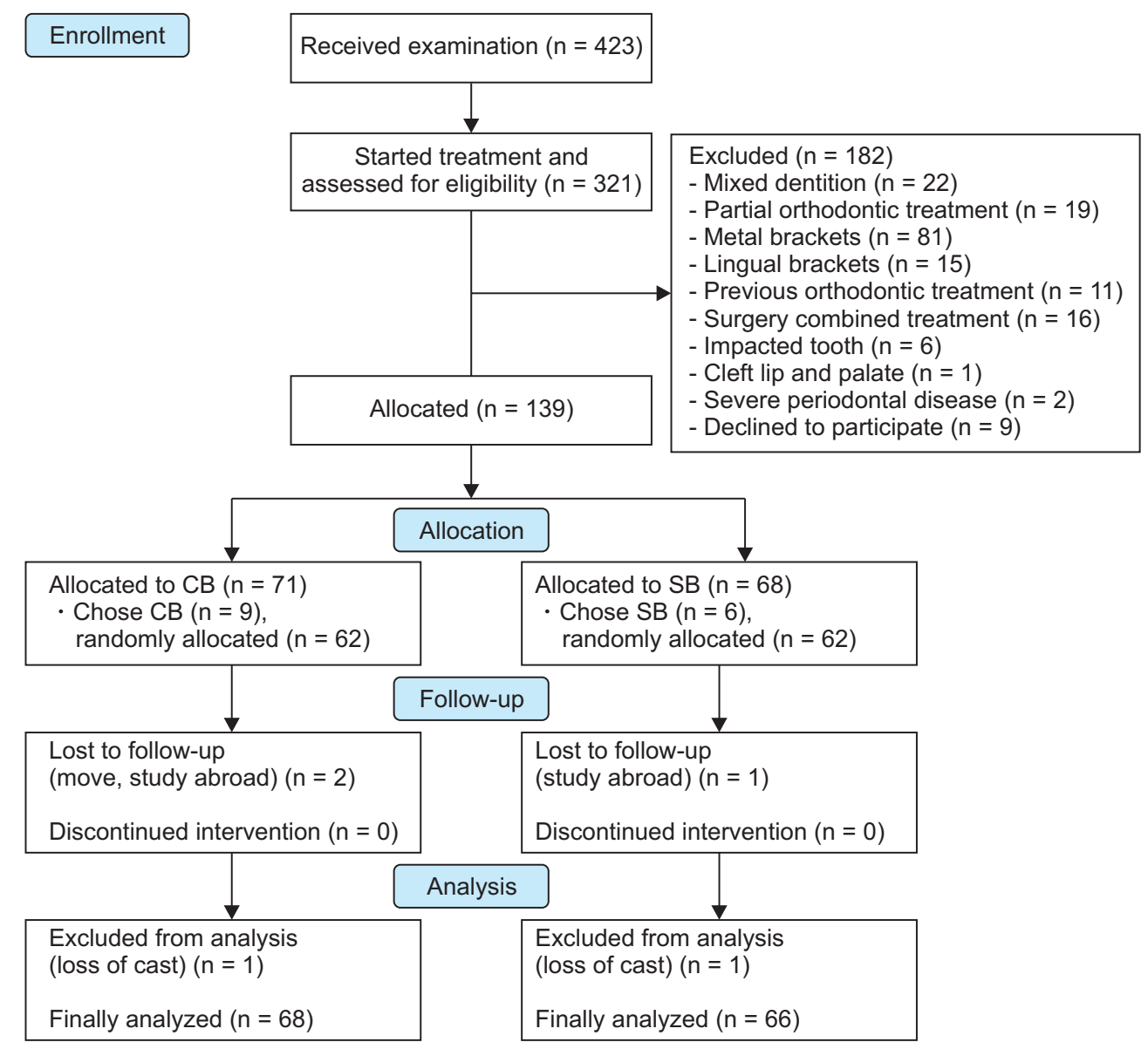

Figure 3. Flow chart of this study.

$\mathrm{CB}$, conventional bracket; $\mathrm{SB}$, self-ligating bracket. 
odontic treatment. From these, 148 met the inclusion criteria, and 139 were distributed into the two groups (Figure 3). Five patients were excluded from the final data; thus, 68 patients in the CB group and 66 in the SB group were eventually analyzed.

Nineteen patients used additional appliances; these included four with Forsus appliances, three with headgear, and two with palatal expanders in the SB group and six with Forsus appliances, one with both an expander and headgear, two with headgear, and one with an expander in the $\mathrm{CB}$ group. Ceramic bracket fracture only occurred in three patients in each group and was not used as a covariate.

The baseline characteristics and clinical features of patients in the CB and SB groups are shown in Tables 1 and 2. The average age was 22.73 years, and the average CRE score was 14.25. Reliability tests for ABO DI, ALD, and CRE using ICC revealed strong intraexaminer reliability (ABO Dl, 0.997; ALD-maxilla, 0.995; ALD-mandible, 0.997; CRE, 0.993). The average treatment duration was 28.63 months, the extraction rate was $71.6 \%$, and OMls were used in $70.1 \%$ of the patients.

Among the nominal variables, extraction and additional appliance use showed significant differences in the $t$-test (Table 3). In the correlation analysis, age, poor elastic wear, and ALD-mandible showed significant correlations (Table 4). When the effects of these five confounding variables were covaried out, the effect of the bracket type on treatment outcomes was significant in ANCOVA (Table 5). Among the eight criteria of CRE, buccolingual inclination, occlusal relation, and root angulation showed significant differences in the MannWhitney $U$ test (Table 6). The models used in stepwise regression analysis are shown in Table 7 . From the four models, model 4 could explain only $25.2 \%$ of the variance in the CRE score; extraction, SB use, poor elastic wear, and additional appliance use decreased the score by $2.307,2.500,1.180$, and 2.244 , respectively.

\section{DISCUSSION}

Increased clinical experience does not automatically improve the CRE score. Even an ABO-certified orthodontist may score as high as $32.21,{ }^{13}$ while orthodontic residents may show average scores of $22.11 .{ }^{17} \mathrm{In}$ regard to this, a study found no significant differences between private practices and orthodontic departments at universities. ${ }^{6}$ It is obvious that if an orthodontist applies consistent efforts (such as the use of prefinished casts and panoramic radiographs) to achieve better CRE scores, the

Table 1. Baseline characteristics of the subjects in the CB and SB groups

\begin{tabular}{|c|c|c|c|}
\hline Variable & CB & SB & Overall \\
\hline Age (yr) & $22.99 \pm 9.09$ & $22.45 \pm 7.78$ & $22.73 \pm 8.44$ \\
\hline \multicolumn{4}{|l|}{ Sex } \\
\hline Male & $22(32.4)$ & $15(22.7)$ & $37(27.6)$ \\
\hline Female & $46(67.6)$ & $51(77.3)$ & $97(72.4)$ \\
\hline Total & 68 & 66 & 134 \\
\hline \multicolumn{4}{|c|}{ Angle classification } \\
\hline I & $35(51.5)$ & $33(50.0)$ & $68(50.7)$ \\
\hline II & $28(41.2)$ & $28(42.4)$ & $56(41.8)$ \\
\hline III & $5(7.4)$ & $5(7.6)$ & $10(7.5)$ \\
\hline ABO DI & $21.40 \pm 10.17$ & $22.23 \pm 12.37$ & $21.81 \pm 11.27$ \\
\hline $\mathrm{DI}<10$ & $6(8.8)$ & $6(9.1)$ & $12(9.0)$ \\
\hline $10 \leq \mathrm{DI}<20$ & $28(41.2)$ & $27(40.9)$ & $55(41.0)$ \\
\hline $20 \leq \mathrm{DI}$ & $34(50.0)$ & $33(50.0)$ & $67(50.0)$ \\
\hline \multicolumn{4}{|c|}{ Irregularity index (mm) } \\
\hline Maxilla & $5.25 \pm 4.34$ & $5.96 \pm 5.11$ & $5.60 \pm 4.73$ \\
\hline Mandible & $3.92 \pm 2.88$ & $4.89 \pm 3.49$ & $4.40 \pm 3.23$ \\
\hline \multicolumn{4}{|c|}{ Arch length discrepancy (mm) } \\
\hline Maxilla & $3.67 \pm 3.73$ & $4.38 \pm 4.23$ & $4.02 \pm 3.99$ \\
\hline Mandible & $3.45 \pm 3.16$ & $3.48 \pm 4.38$ & $3.47 \pm 3.80$ \\
\hline
\end{tabular}

Values are presented as mean \pm standard deviation or number (\%).

$\mathrm{CB}$, conventional bracket; SB, self-ligating bracket; ABO DI, American Board of Orthodontics Discrepancy Index. 
Table 2. Clinical variables of the CB and SB groups

\begin{tabular}{lccc}
\hline \multicolumn{1}{c}{ Variable } & CB & SB & Overall \\
\hline Treatment duration (mo) & $29.25 \pm 7.53$ & $27.98 \pm 7.10$ & $28.63 \pm 7.32$ \\
Extraction & $49(72.1)$ & $47(71.2)$ & $96(71.6)$ \\
Four premolar extraction & $36(52.9)$ & $34(51.5)$ & $70(52.2)$ \\
OMI use & $46(67.6)$ & $48(72.7)$ & $94(70.1)$ \\
Ave OMI & 3.70 & 4.02 & 3.86 \\
OMI failure & $0.63 \pm 0.85$ & $0.87 \pm 1.30$ & $0.75 \pm 1.10$ \\
Additional appliance & $10(14.7)$ & $9(13.6)$ & $19(14.2)$ \\
Bracket failure & $1.81 \pm 1.93$ & $1.12 \pm 1.09$ & $1.47 \pm 1.61$ \\
Poor elastic wear & $2.12 \pm 2.47$ & $1.77 \pm 1.94$ & $1.96 \pm 2.23$ \\
Poor oral hygiene & $3.10 \pm 4.51$ & $3.15 \pm 3.08$ & $3.13 \pm 3.86$ \\
Missed appointment & $1.87 \pm 3.58$ & $1.73 \pm 3.69$ & $1.80 \pm 3.62$ \\
Total CRE score & $12.90 \pm 4.62$ & $15.64 \pm 4.81$ & $14.25 \pm 4.90$ \\
CRE $<10$ & $13(19.1)$ & $6(9.1)$ & $19(14.2)$ \\
$10 \leq$ CRE $<20$ & $48(70.6)$ & $46(69.7)$ & $94(70.1)$ \\
$20 \leq$ CRE $<30$ & $7(10.3)$ & $14(21.2)$ & $21(15.7)$ \\
\hline
\end{tabular}

Values are presented as mean \pm standard deviation, number (\%), or number only.

$\mathrm{CB}$, conventional bracket; SB, self-ligating bracket; OMI, orthodontic mini-implant; OMI use, number of patients who received OMIs; Ave OMI, total number of OMIs used/total number of subjects who used OMIs; OMI failure, total number of failed OMIs/total number of subjecs who used OMIs; CRE, Cast-Radiograph Evaluation score.

Table 3. Comparison of the CRE score (after square root transforamtion) for the nominal variables

\begin{tabular}{llllccc}
\hline \multicolumn{1}{c}{ Variable } & Group & n & CRE & CRE-SR & t-value & p-value \\
\hline Sex & Male & 37 & 14.11 & $3.76 \pm 0.80$ & 0.362 & 0.719 \\
& Female & 97 & 13.72 & $3.70 \pm 0.59$ & & \\
Extraction & Nonextraction & 38 & 16.14 & $4.02 \pm 0.56$ & 3.500 & $0.001^{* *}$ \\
\multirow{2}{*}{ OMI use } & Extraction & 96 & 12.96 & $3.60 \pm 0.65$ & & \\
& Yes & 94 & 13.37 & $3.66 \pm 0.61$ & -1.730 & 0.086 \\
Additional appliance & No & 40 & 14.95 & $3.87 \pm 0.72$ & & -3.292 \\
& Yes & 19 & 17.28 & $4.16 \pm 0.65$ & $0.001^{* *}$ \\
& No & 115 & 13.29 & $3.65 \pm 0.62$ & & \\
\hline
\end{tabular}

Values are presented as number only or mean \pm standard deviation.

Independent $t$-tests were performed.

CRE, Cast-Radiograph Evaluation score; CRE-SR, CRE after square root transformation; OMI, orthodontic mini-implant.

** $p<0.01$.

score will improve. ${ }^{17,18}$

The average CRE score in the present study was 14.25 , which is much lower than that in a recent systematic review (27.9). ${ }^{6}$ There are several possible reasons for this discrepancy.

A recent systematic review showed that extraction of four premolars was associated with an improved CRE score of 4.9 points. $^{6}$ A poorer CRE score is observed in cases of nonextraction treatment because expansion or molar distalization may result in inappropriate torque or angulation of the posterior teeth in some cases. The extraction rate in this study was $71.6 \%$, which was higher than that in previous studies $(24.3-34.4 \%)^{7,8,18}$ and may have played a role in lowering the CRE score.

People may behave differently when they are aware that they are being observed. Therefore, the "Hawthorne effect" may be an important factor affecting the generalizability of clinical research findings to routine practice. ${ }^{19}$ The present study was a prospective one, where the operator knew that the patients' treatment results 
would be qualitatively evaluated. Accordingly, the CRE score may have been affected by the Hawthorne effect. Correlation analysis showed no significant correlation between the CRE score and treatment duration; however, considering the significantly longer treatment period and lower CRE score than those in other studies, it is possible to speculate that more time and effort were invested to lower the mean CRE score. The treatment duration was $>3$ years for 18 patients. A longer treatment period increases the likelihood of decalcification;

Table 4. Correlation between continuous variables and the Cast-Radiograph Evaluation score (after square root transformation)

\begin{tabular}{lcc}
\hline \multicolumn{1}{c}{ Variable } & $\begin{array}{c}\text { Correlation } \\
\text { coefficient }\end{array}$ & p-value \\
\hline Age & -0.213 & $0.014^{*}$ \\
Treatment duration (log) & 0.000 & 0.998 \\
OMI failure & -0.121 & 0.163 \\
Bracket failure & 0.077 & 0.376 \\
Poor elastic wear & 0.244 & $0.005^{* *}$ \\
Poor oral hygiene & 0.131 & 0.132 \\
Missed appointment & 0.112 & 0.198 \\
ABO DI & 0.088 & 0.310 \\
ALD-maxilla & -0.010 & 0.908 \\
ALD-mandible & -0.171 & $0.048^{*}$ \\
\hline
\end{tabular}

Pearson's correlation analysis was performed.

OMI, orthodontic mini-implant; OMI failure, total number of failed OMIs/number of patients who received OMIs; ABO DI, American Board of Orthodontics Discrepancy Index; ALD, arch length discrepancy; ALD-maxilla, arch length discrepancy of maxilla; ALD-mandible, arch length discrepancy of mandible.

${ }^{*} p<0.05 ;{ }^{* *} p<0.01$. therefore, a long treatment period is usually not desirable. $^{20}$

Although a study showed that treatment outcomes worsen when the treatment duration is long, ${ }^{18}$ the effect of the treatment duration does not seem to be significant according to a systematic review $;{ }^{6}$ moreover, no significant effect was found in the present study. The longer treatment duration (28.63 months) relative to that found in a recent systematic review $(20.02$ months $)^{21}$ may have been influenced by the high extraction rate and the aforementioned Hawthorne effect.

The effects of case complexity on the quality of treatment are controversial. Some studies have shown results implying that case difficulty is not significantly correlated with treatment outcomes, ${ }^{9,10}$ while others have shown the two to be significantly correlated, although the correlation coefficients were only 0.17 and 0.20 , respectively. ${ }^{11,12} \mathrm{ABO}$ DI was developed to evaluate the pretreatment case complexity, ${ }^{22}$ and the value in this study

Table 5. Influence of the bracket type after removal of the effects of covariates on the Cast-Radiograph Evaluation score (after square root transformation)

\begin{tabular}{lrl}
\hline \multicolumn{1}{c}{ Variable } & \multicolumn{1}{c}{ F } & p-value \\
\hline Bracket type & 17.579 & $<0.001^{* * *}$ \\
Covariates & & \\
Extraction & 7.646 & 0.007 \\
Age & 0.245 & 0.621 \\
Poor elastic wear & 10.445 & 0.002 \\
ALD-mandible & 2.039 & 0.156 \\
Additional appliance & 2.906 & 0.091 \\
\hline
\end{tabular}

Analysis of covariance was performed.

ALD-mandible, arch length discrepancy of mandible. *** $p<0.001$.

Table 6. Comparison of the Cast-Radiograph Evaluation criteria between the CB and SB groups

\begin{tabular}{llcc}
\hline \multicolumn{1}{c}{ Variable } & CB & SB & p-value \\
\hline Alignment \& rotation & $0.84(0-4)$ & $1.18(0-4)$ & 0.059 \\
Marginal ridges & $0.99(0-4)$ & $0.97(0-3)$ & 0.867 \\
Buccolingual inclination & $2.75(0-6)$ & $4.26(0-9)$ & $<0.001^{* * *}$ \\
Overjet & $1.01(0-4)$ & $0.77(0-5)$ & 0.144 \\
Occlusal contacts & $4.97(0-12)$ & $5.35(0-11)$ & 0.229 \\
Occlusal relationships & $1.85(0-14)$ & $2.29(0-11)$ & $0.038^{*}$ \\
Interproximal contacts & $0.00(0-0)$ & $0.03(0-2)$ & 0.310 \\
Root angulation & $0.53(0-4)$ & $0.83(0-3)$ & $0.010^{*}$ \\
\hline
\end{tabular}

Values are presented as mean (minimum-maximum).

Mann-Whitney $U$ test was performed.

$\mathrm{CB}$, conventional bracket; SB, self-ligating bracket.

${ }^{*} p<0.05$; ${ }^{* * *} p<0.001$. 
Table 7. Results of stepwise regression analysis for prediction of the Cast-Radiograph Evaluation score (after square root transformation)

\begin{tabular}{ccccc}
\hline Model & $\mathbf{R}$ & $\mathbf{R}^{2} /$ adjusted $\mathbf{R}^{2}$ & $\mathbf{F}$ & $\boldsymbol{p}$-value \\
\hline 1 & 0.291 & $0.085 / 0.078$ & 12.250 & $0.001^{* *}$ \\
2 & 0.408 & $0.166 / 0.153$ & 13.049 & $<0.001^{* * *}$ \\
3 & 0.491 & $0.241 / 0.223$ & 13.743 & $<0.001^{* * *}$ \\
4 & 0.524 & $0.274 / 0.252$ & 12.176 & $<0.001^{* * *}$ \\
\hline
\end{tabular}

Model 1, $-0.418 \times$ extraction +4.018 ; Model 2, $-0.414 \times$ extraction $+0.369 \times$ bracket type $+3.834 ;$ Model $3,-0.423 \times$ extraction $+0.396 \times$ bracket type $+0.080 \times$ poor elastic wear +3.671 ; Model $4,-0.363 \times$ extraction $+0.398 \times$ bracket type $+0.072 \times$ poor elastic wear $+0.351 \times$ additional appliance use +3.593 .

${ }^{* *} p<0.01 ; * * 0<0.001$.

(21.81) was much higher than that in previous studies $(15.49-16.8) ;^{12,17,23}$ furthermore, it showed no significant correlation with the CRE score.

Although systematic reviews have found no significant differences in treatment outcomes between SBs and $\mathrm{CBs}^{3,4}$ ANCOVA in the present study revealed a statistically significant difference. However, the value of the difference was only 2.74 , so its clinical significance is questionable. Among the CRE measurements, buccolingual inclination, occlusal relation, and root angulation showed significant differences, and further research is needed to determine if these differences were caused by the distance between the archwire and tooth surface. ${ }^{5}$ The fact that all the patients were mixed and treated together may have masked the strengths or weaknesses of SBs.

In the present study, age was associated with treatment outcomes, probably because older patients cooperate better than do younger patients. ${ }^{24}$ However, considering the correlation coefficient was only -0.213 , the influence seems to be limited.

Previous studies showed that factors related to patient cooperation increased the treatment duration. ${ }^{7,8,25}$ In the present study, only poor elastic wear showed a statistically significant correlation with the CRE score. When considering the effects of these factors on treatment outcomes, it is assumed that poor elastic wear is more directly related to treatment outcomes than are missed appointments, bracket failure, and poor oral hygiene.

In the present study, all patients with post-treatment data, including those with premature termination of treatment, were included in the analysis. The number of patients with premature termination of treatment was only three. Even if treatment was continued for these patients, it would have ended within 3-4 months, so the impact of their data on the study results is considered negligible.

Although their influence was not analyzed in this study, the diversity of the tooth morphology ${ }^{26}$ and the size ratio of the maxillary and mandibular teeth ${ }^{27}$ can affect the treatment outcome. While tooth reshaping, archwire bending, or interproximal stripping may reduce the impact of these factors, the effects of anatomical diversity cannot be completely eliminated in many cases. Therefore, deduction of a few points from the CRE score seems inevitable. Because several different variables can have an effect, the predictability through stepwise regression analysis was low in this study.

OMl-related factors did not appear to have a significant impact on the treatment outcome, the primary reason being that most $\mathrm{OMI}$ cases involved simple anchorage reinforcement. As it becomes increasingly difficult to obtain cooperation from adolescents, and with systematic reviews showing that the long-term orthopedic effects of headgear are not significant, ${ }^{28,29}$ headgear was recommended only if the patient or parents refused to receive OMls.

Additional appliances may have affected the torque or angulation of the posterior teeth. Because a small number of patients used an additional appliance, and because several appliances were used, it was difficult to analyze the effects of each additional appliance on treatment outcomes in the present study.

A recent study showed that custom indirect bonding using CAD/CAM technology could reduce the treatment duration while providing excellent treatment outcomes. ${ }^{30}$ It seems that precise bracket positioning can affect the effectiveness and efficiency of treatment, and further research on this subject is required.

\section{Limitations}

Because this study included patients treated by a single orthodontist in a single private clinic, all showed the same racial and cultural backgrounds. Accordingly, generalization of our results requires caution.

\section{CONCLUSION}

In this prospective cohort study, CBs exhibited better treatment outcomes than did SB. However, the differ- 
ence was small, so its clinical significance seems to be limited. Extraction, SB use, poor elastic wear, and additional appliance use may have a significant impact on treatment outcomes.

\section{CONFLICTS OF INTEREST}

No potential conflict of interest relevant to this article was reported.

\section{REFERENCES}

1. Casko JS, Vaden JL, Kokich VG, Damone J, James $\mathrm{RD}$, Cangialosi $\mathrm{TJ}$, et al. Objective grading system for dental casts and panoramic radiographs. American Board of Orthodontics. Am J Orthod Dentofacial Orthop 1998;114:589-99.

2. Onyeaso CO, Begole EA. Relationship between index of complexity, outcome and need, dental aesthetic index, peer assessment rating index, and American Board of Orthodontics objective grading system. Am J Orthod Dentofacial Orthop 2007;131:248-52.

3. Papageorgiou SN, Konstantinidis 1, Papadopoulou K, Jäger A, Bourauel C. Clinical effects of pre-adjusted edgewise orthodontic brackets: a systematic review and meta-analysis. Eur J Orthod 2014;36:350-63.

4. Chen SS, Greenlee GM, Kim JE, Smith CL, Huang GJ. Systematic review of self-ligating brackets. Am J Orthod Dentofacial Orthop 2010;137:726.e1-726. e18; discussion 726-7.

5. Bennett JC. Fundamentals of orthodontic bracket selection: a user guide. London: Le Grande Pub.; 2010.

6. Papageorgiou SN, Höchli D, Eliades T. Outcomes of comprehensive fixed appliance orthodontic treatment: a systematic review with meta-analysis and methodological overview. Korean J Orthod 2017;47:401-13.

7. Beckwith FR, Ackerman RJ Jr, Cobb CM, Tira DE. An evaluation of factors affecting duration of orthodontic treatment. Am J Orthod Dentofacial Orthop 1999;115:439-47.

8. Skidmore KJ, Brook KJ, Thomson WM, Harding WJ. Factors influencing treatment time in orthodontic patients. Am J Orthod Dentofacial Orthop 2006;129:230-8.

9. Chaison ET, Liu X, Tuncay OC. The quality of treatment in the adult orthodontic patient as judged by orthodontists and measured by the Objective Grading System. Am J Orthod Dentofacial Orthop 2011;139(4 Suppl):S69-75.

10. Cansunar HA, Uysal T. Relationship between pretreatment case complexity and orthodontic clinical outcomes determined by the American Board of Or- thodontics criteria. Angle Orthod 2014;84:974-9.

11. Campbell CL, Roberts WE, Hartsfield JK Jr, Qi R. Treatment outcomes in a graduate orthodontic clinic for cases defined by the American Board of Orthodontics malocclusion categories. Am J Orthod Dentofacial Orthop 2007;132:822-9.

12. Pulfer RM, Drake CT, Maupome G, Eckert GJ, Roberts WE. The association of malocclusion complexity and orthodontic treatment outcomes. Angle Orthod 2009;79:468-72.

13. Djeu G, Shelton C, Maganzini A. Outcome assessment of Invisalign and traditional orthodontic treatment compared with the American Board of Orthodontics objective grading system. Am J Orthod Dentofacial Orthop 2005;128:292-8; discussion 298.

14. Jung MH. A comparison of second premolar extraction and mini-implant total arch distalization with interproximal stripping. Angle Orthod 2013;83:6805.

15. Johnson G, Walker MP, Kula K. Fracture strength of ceramic bracket tie wings subjected to tension. Angle Orthod 2005;75:95-100.

16. Silness J, Loe H. Periodontal disease in pregnancy. 11. Correlation between oral hygiene and periodontal condition. Acta Odontol Scand 1964;22:121-35.

17. Brown PN, Kulbersh R, Kaczynski R. Clinical outcomes assessment of consecutively finished patients in a 24-month orthodontic residency: a 5-year perspective. Am J Orthod Dentofacial Orthop 2011;139:665-8.

18. Knierim K, Roberts WE, Hartsfield J Jr. Assessing treatment outcomes for a graduate orthodontics program: follow-up study for the classes of 2001-2003. Am J Orthod Dentofacial Orthop 2006;130:648-55, 655.e1-3.

19. McCarney R, Warner J, lliffe S, van Haselen R, Griffin M, Fisher P. The Hawthorne Effect: a randomised, controlled trial. BMC Med Res Methodol 2007;7:30.

20. Knösel M, Klang E, Helms HJ, Wiechmann D. Occurrence and severity of enamel decalcification adjacent to bracket bases and sub-bracket lesions during orthodontic treatment with two different lingual appliances. Eur J Orthod 2016;38:485-92.

21. Tsichlaki A, Chin SY, Pandis N, Fleming PS. How long does treatment with fixed orthodontic appliances last? A systematic review. Am J Orthod Dentofacial Orthop 2016;149:308-18.

22. Cangialosi TJ, Riolo ML, Owens SE Jr, Dykhouse VJ, Moffitt AH, Grubb JE, et al. The ABO discrepancy index: a measure of case complexity. Am J Orthod Dentofacial Orthop 2004;125:270-8.

23. Brown MW, Koroluk L, Ko CC, Zhang K, Chen M, 
Nguyen T. Effectiveness and efficiency of a CAD/ CAM orthodontic bracket system. Am J Orthod Dentofacial Orthop 2015;148:1067-74.

24. Jung MH. Survival analysis of brackets and tubes: a twelve-month assessment. Angle Orthod 2014; 84:1034-40.

25. Bukhari OM, Sohrabi K, Tavares M. Factors affecting patients' adherence to orthodontic appointments. Am J Orthod Dentofacial Orthop 2016;149:319-24.

26. Kim HK, Kwon HB, Hyun HK, Jung MH, Han SH, Park YS. Submorphotypes of the maxillary first molar and their effects on alignment and rotation. Am J Orthod Dentofacial Orthop 2014;146:286-93.

27. Bolton WA. The clinical application of a tooth-size analysis. Am J Orthod 1962;48:504-29.
28. Dermaut LR, Aelbers CM. Orthopedics in orthodontics: fiction or reality. A review of the literature--part 11. Am J Orthod Dentofacial Orthop 1996;110:66771.

29. Batista KB, Thiruvenkatachari B, Harrison JE, O'Brien KD. Orthodontic treatment for prominent upper front teeth (Class 11 malocclusion) in children and adolescents. Cochrane Database Syst Rev 2018; 3:CD003452.

30. Moreira FC, Vaz LG, Guastaldi AC, English JD, Jacob HB. Potentialities and limitations of computer-aided design and manufacturing technology in the nonextraction treatment of Class 1 malocclusion. Am J Orthod Dentofacial Orthop 2021;159:86-96. 